\title{
Development of Quantitative In Situ TEM Nanomechanical Testing for Polymers
}

\author{
Nathan Velez ${ }^{1,2}$, Frances Allen ${ }^{1,2}$, Mary Ann Jones ${ }^{3}$, Gregory Meyers ${ }^{3}$ and Andrew M. Minor ${ }^{1,2}$ \\ 1. Department of Materials Science and Engineering, UC Berkeley, Berkeley, CA \\ 2. National Center for Electron Microscopy, Molecular Foundry, LBNL, Berkeley, CA \\ 3. Core R\&D - Analytical Sciences, The Dow Chemical Company, Midland, MI
}

The recent development of in situ Transmission Electron Microscopy (TEM) nanomechanical testing techniques has mostly benefitted our understanding of fundamental deformation mechanisms in hard materials such as metals and ceramics [1]. Here, we report on recent progress extending these techniques to study polymeric materials using an in situ TEM nanoindenter with a Push-to-Pull (PTP) device to perform quantitative tensile tests on thin polymer sheets.

Two different polymer systems were used to demonstrate the advantages of this method. The first was copolymer spherical particles with radii on the order of hundreds of microns (Figure 1a), too small for traditional bulk tensile testing. The second is a $\sim 300 \mu \mathrm{m}$ thick co-extruded sheet of a polymer multilayer with in-plane interfaces spaced $\sim 1 \mu \mathrm{m}$ apart. Traditional tensile testing techniques are restricted to straining the specimen along the direction of the interface, whereas the method described here allows for the interface to be arbitrarily oriented with respect to the direction of applied force. This enables the direct testing of the interfacial strength while observing the dynamics of the deformation in-situ.

The general procedure for preparing a specimen is a two-part process. First, thin sections of the polymer are deposited onto the PTP device using an ultramicrotome (Figure 1b). The copolymer spheres are hydrophobic and the glass transition temperature $\left(\mathrm{T}_{\mathrm{g}}\right)$ is well above room temperature. This allows 'wet' sectioning to be performed at room temperature; floating the sections onto a water reservoir. A section can then be placed directly onto the PTP device using a loop tool. The multilayered polymer has $\mathrm{T}_{\mathrm{g}}$ 's that are lower than room temperature and therefore requires cryo-ultramicrotomy to obtain sections.

The second stage of specimen preparation utilizes a focused ion beam (FIB) to micromachine a tensile gauge (dogbone) to clear any material overlapping the gaps on the PTP device (Figure 1c). The dimensions of the dogbones are scaled down versions of ATSM standards to avoid stress concentrators that would cause fracture in undesired locations. Care must be taken to avoid excessive damage from the ion beam milling by utilizing low currents or milling at cryogenic temperatures. Finally, an accurate measurement of the section thickness can be obtained via atomic force microscopy (AFM). Following sample preparation and analysis, the in situ experiments are performed using a Hysitron PI 95 TEM Picoindenter in a JEOL 3010 TEM.

Our initial results demonstrate that it is possible to both measure the deformation of the nano-tensile samples of polymers quantitatively as well as observe the deformation characteristics in situ. In addition to presenting these results, we will address our efforts to minimize and mitigate potential artifacts from the ion beam during gauge milling and the electron beam during imaging [2]. 


\section{References:}

[1] Q. Yu, M. Legros, A.M. Minor, MRS Bulletin 40 (2015), p. 62.

[2] Work at the Molecular Foundry at Lawrence Berkeley National Laboratory was supported by the U.S. Department of Energy under Contract \# DE-AC02-05CH11231

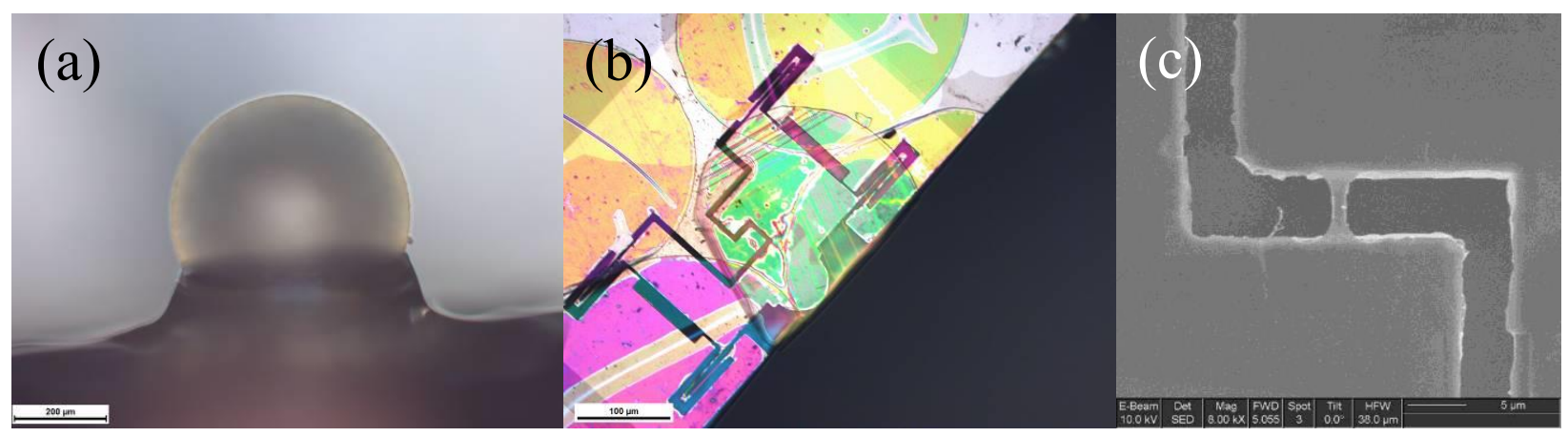

Figure 1. (a) Spherical copolymer particle mounted on an ultramicrotome stub. (b) Copolymer sections deposited with a loop tool onto a PTP device. (c) SEM image of milled dogbone specimen and cleared gaps of PTP device. 